\title{
Use of Temporalis Myofascial Flap in Maxillary Reconstruction
}

\author{
${ }^{1}$ Choubarga Nayak, ${ }^{2}$ Santosh Kumar Swain
}

\begin{abstract}
Maxillary ameloblastoma is a benign odontogenic neoplasm. Excision should involve wide margins because of its high recurrence rate and ideally be followed by reconstruction. A 23 year-old female patient presented with recurrent ameloblastoma. One year previously, she had undergone a curettage excision. The recurrent lesion was managed by a total maxillectomy preserving the inferior orbital rim. Simultaneous reconstruction was performed with an temporalis myofascial flap for obliteration of the maxillary sinus and nasal cavities. Reconstruction of mid facial defects after tumor resection or trauma has evolved from skin graft to pedicled flaps and more recently, to free tissue transfer. Each of these reconstructive modalities has its specific indications, advantages and drawbacks. Temporalis muscle is one of the regional flaps that can be used to reconstruct midfacial defects. The muscle is available close to the surgical field and has a constant vascular supply. However, the flap has a limited arc of rotation and most of the muscle bulk is used in the pedicle. Therefore, various modifications of the surgical procedure were attempted in order to increase the arc of rotation of the flap. The temporalis muscle flap can provide a reliable, one-stage reconstructive alternative for more complicated surgical procedures for moderate-sized midfacial defects. Dimensions and arc of rotation of the flap may be increased by using temporalis muscle and deep temporal fascia as a myofascial unit.
\end{abstract}

Keywords: Ameloblastoma, Temporalis myofascial flap, Maxillectomy.

How to cite this article: Nayak C, Swain SK. Use of Temporalis Myofascial Flap in Maxillary Reconstruction. Clin Rhinol An Int J 2014;7(2):73-75.

\section{Source of support: Nil}

Conflict of interest: None declared

\section{INTRODUCTION}

Ameloblastoma is a benign odontogenic neoplasm of the mandible and maxilla. ${ }^{1,2}$ However, it is reported that

\footnotetext{
${ }^{1}$ Assistant Professor, ${ }^{2}$ Associate Professor

${ }^{1}$ Department of Maxillofacial Surgery, vss Medical College Burla, Odisha, India

${ }^{2}$ Department of ENT, Institute of Medical Sciences and SUM Hospital, Bhubaneshwar, Odisha, India

Corresponding Author: Santosh Kumar Swain, Associate Professor, Department of ENT, Institute of Medical Sciences and SUM Hospital, Bhubaneshwar, Odisha, India, Phone: 06742384166, e-mail: swainsantoshbbsr@yahoo.com
}

maxillary ameloblastoma behave more aggressively and have a poorer prognosis compared with mandibular ameloblastoma. ${ }^{3}$ The painless and slow growth of the lesion and the thin bone of the maxilla are the main factors involved in delay in recognizing a maxillary ameloblastoma. ${ }^{4}$ Conservative treatment of an ameloblastoma usually results in recurrence and possible malignant transformation. ${ }^{5}$ Radical excision of the tumor followed by adequate reconstruction can improve survival and provide more satisfactory functional and cosmetic results. ${ }^{6}$ However, reconstruction of multifaceted maxillary defects is a challenge to thereconstructive surgeon, as it requires provision of adequate anatomical structural support to separate the oral and nasal cavities, and to obliterate the dead space in the maxillary sinus. Here, we report on a recurrent maxillary ameloblastoma that was successfully treated with radical excision and simultaneous reconstruction using temporomyofascial flap for the maxillary antrum, oral and nasal cavities. ${ }^{15}$

\section{CASE REPORT}

A 23-year-old female patient presented with a right upper molar gingival swelling that had been present for several months. She noted unusual loosening of her right first upper molar tooth, which was extracted by a local dentist. However, the wound did not heal and she was eventually referred to a Surgeon. Under general anesthesia, the patient underwent curettage via an intraoral approach. After surgery, the final histopathology showed plexiform ameloblastoma. The wound remained unhealed with a persistent oroantral fistula, which was managed by secondary closure. The patient then remained symptom-free for 2 years. However, due to persistent right facial swelling and numbness, the patient reported to us. After clinical examination revealed a mass about $6 \times 5 \mathrm{~cm}$ in size in the left upper alveolar region and an incisional biopsy was performed. The pathology report cited ameloblastoma of maxilla. A computed tomography (CT) scan was requested, which revealed a space-occupying lesion in the maxillary sinus involving the anterior wall of the maxillary sinus and nose (Figs 1A and B), but orbit was spared. A recurrent ameloblastoma was suspected and radical excision of the lesion was planned. The patient then underwent a total maxillectomy via a Weber-Ferguson incision with preservation of the orbit. The patient was taken 

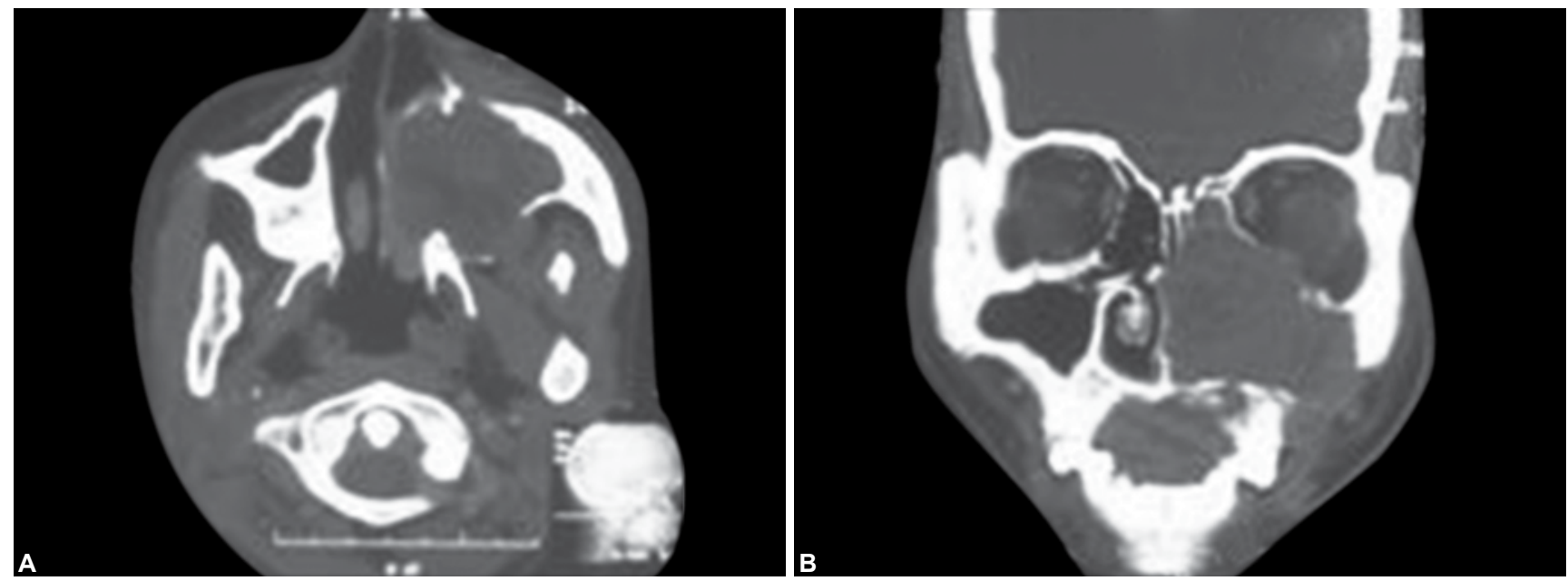

Figs $1 \mathrm{~A}$ and $\mathrm{B}$ : Computed tomography (CT) showing a space-occupying lesion in the maxillary sinus involving the anterior wall of the maxillary sinus and nose

for primary reconstruction of maxillary defects with a temporalis myofascial flap.

\section{SURGICAL TECHNIQUE}

The temporalis muscle was exposed through a coronal incision. The technique of flap elevation and transposition is the same as described by Wolfe. ${ }^{14}$ The zygomatic arch and the coronoid process were divided as the flap was used to cover maxillectomy defects. The two laminas of the deep temporal fascia were separate from the zygomatic arch up to the area of fusion. Attachment of the deep temporal fascia to the muscle was reinforced by few absorbable sutures, and the muscle is covered with a skin graft. The temporalis muscle is then used to reconstruct the oral cavity. The entire muscle is rotated into the infratemporal region, on to the size of the palatal defect. Deep sutures are used to secure the flap, and resorbable sutures are placed intraorally to oppose the flap and the remaining palatal mucosa. Any remaining posterior temporalis muscle is secured to the lateral orbital rim to fill the temporal fossa. The scalp incision is closed.

\section{DISCUSSION}

Maxillary ameloblastoma is an aggressive benign tumor. Rapid growth of the tumor is not unusual due to its closeness to the maxillary sinus. However, the patient may not notice the mass until has eaten away the bone. Although histologically benign, recurrence rate is high. Occasionally it transforms into ameloblastic carcinoma and metastasizes to distant organs, most commonly the lungs. ${ }^{7}$ Repeated surgical treatment has a potential of spreading lesions to the base of the skull, which can be fatal. Therefore, radical resection of the tumor at the first surgery is important to achieve a complete cure. Due to the high recurrence rate of maxillary ameloblastoma, some authors suggest using only an obturator for the defect left after tumor resection in order to retain a better view of the site. ${ }^{8,9}$ There is still a great deal of controversy regarding the primary reconstruction of maxillectomy defects. ${ }^{10,11}$ Maxillectomy defects reconstructed with a vascularized iliac crest bone graft with internal oblique muscle pedicle from the deep circumflex iliac artery can have up to a $38 \%$ mortality following grafting mainly due to recurrent disease. This is because once the patient has been grafted and oral cavity is sealed from the resection cavity, it is extremely difficult to detect the recurrence. However, the obturator cannot replace all deficiencies in the maxillary bone, and this can result in midfacial depression. Furthermore, the poor stability of a palatal obturator, due to lack of soft and hard tissue support and anchorage, will be a problem for the patient in daily life. For a young female patient, reconstruction, which takes into consideration concerns of both function and appearance, is important. A one-stage complete reconstruction after radical excision, if possible, is the ideal approach.

A large variety of techniques are available for reconstruction of midfacial defects. Each technique has its advantages and limitations. Traditional reconstruction included skin grafting and placement of a prosthesis for maxillary defect, and spontaneous granulation or epithelialization of orbital exenteration defect. However, the healing time is prolonged, the cosmetic outcome is poor, and there is a possibility of developing a fistula between the oral cavity or the orbit and the nasal cavity or the paranasal sinuses. There are several methods that can be used to reconstruct the maxilla defect after excision of the tumor: which include a prosthetic obturator, temporalis myofascial flap. Myocutaneous free flaps, with or without an osseous component, have also been described to reconstruct the palate. Use of the temporalis myofascial rotational flap has allowed expeditious treatment and rapid return of function, thereby maximizing the quality of life for the patient and minimizing the functional and 
cosmetic deficits. Temporalis muscle flap has been criticized because of its short arc of rotation. This is why the flap can cover only moderate sized defects, measuring 4 to $8 \mathrm{~cm}$ in the greatest dimension. ${ }^{12}$ It cannot reach the mid line and most of the muscle bulk is used in the pedicle. ${ }^{13}$ Temporalis muscle is one of the regional flaps that have been used in facial reconstruction. It has a constant vascular supply from the deep temporal and the middle temporal vessels that are located on the deep surface of muscle. ${ }^{15}$ The temporalis muscle can be split safely in the coronal plane, since the blood supply travels in the same direction. ${ }^{14}$

\section{CONCLUSION}

The flap is reliable as long as its vascular supply is preserved and the operative time is short compared with freetissue transfer. Its dimensions and arc of rotation may be increased by using temporalis muscle and deep temporal fascia myofascial unit. Temporalis muscle flap should be taken into consideration before deciding on more extensive reconstructive procedures especially for moderate defects.

\section{REFERENCES}

1. Tamu, Sapp P, Huang C, Abemayor E. Malignant ameloblastoma: classification, diagnostic and therapeutic challenges. J Otolaryngol 2011;93:44-48.

2. Jack. Ameloblastoma of the jaws. Gan To Kagaku Ryoho 2012; 27 (Suppl 2):261-267.
3. John IT, Tapp, Forte RA. An anatomical classification of maxillary ameloblastoma as an aid to surgical treatment. Int $\mathrm{J}$ Craniomaxillofac Surg 2012;24:230-236.

4. Oten, Makos C, Dimitrakopoulos J, Kariki H. Ameloblastoma of the maxilla. Case report. Nez Dent J 2010;44:56-55.

5. Topag AA, Arole G, Adekeye BO. Recurrent ameloblastoma of the jaws. A follow-up study. Int J Craniomaxillofac Surg 2011; 27:421-460.

6. Zolawr RA, Gratz KW. Maxillary ameloblastomas: a review of the literature and of a 15-year database. J Craniomaxillofac Surg 2009;30:276-279.

7. Dogan R, Sungur A. Pulmonary metastasectomy in the treatment of recurrent ameloblastoma of the maxilla and mandible: a case report. Eur Arch Otorhinolaryngol 2009;258:25-27.

8. Tolab G. Use of obturators in rehabilitation of maxillectomy defects. Ann R Australas Coll Dent S 2008;15:75-79.

9. Rogers SN, Lowe D, McNally D, Brown JS, Vaughan ED. Health-related quality of life after maxillectomy: a comparison between prosthetic obturation and free flap. Int J Oral Maxillofac Surg 2013;61(2):174-181.

10. Demirkan F, Chen HC, Wei FC, Chen HH, Jung SG, Hau SP, Liao CT. The versatile anterolateral thigh flap: a musculocutaneous flap in disguise in head and neck reconstruction. $\mathrm{Br}$ J Plast Surg 2010;53:30-36.

11. Foster D, Sin MI, et al. Reconstruction of complex mid facialdefects. Eu Plast Reconstr Surg 2008;99:15-25.

12. Deres A, Maz M, Vada H, Barutcu A. Reverse temporalis muscle flap for the reconstruction of orbital exenteration defect. Int Plast Surg 2012;48:521-526.

13. Antonyshyn O, Gruss JS, Birt BD. Versatility of temporal muscle and fascia flaps. Aus J Plast Surg 2008;41:118-131.

14. Lapu PG, Wole SA. The temporalis muscle flap revisited on its centennial: Advantages, new uses and disadvantages. Int Plast Reconstr Surg 2011;12:998-997.

15. Buli G, Hap W, Ruber H, Freiger G. The temporalis: Blood supply and innervations. J Reconstr Surg 2008;109:1862-1869. 